\title{
Eigen Faces and Principle Component Analysis for Face Recognition Systems: A Comparative Study
}

\author{
Abdelfatah Aref Tamimi ${ }^{1}$, Omaima N. A. AL-Allaf ${ }^{2}, \quad$ Mohammad A. Alia ${ }^{3}$
}

${ }^{1}$ Associate Professor, Dept. of CS, Faculty of Sciences \& IT, Al-Zaytoonah University of Jordan, Amman, Jordan, email: drtamimi@zuj.edu.jo

${ }^{2}$ Assistant Professor, Dept. of Basic Sciences, Faculty of Sciences \& IT, Al-Zaytoonah University of Jordan, Amman, Jordan, email:omaimaalallaf@zuj.edu.jo

${ }^{3}$ Associate Professor, Dept. of CIS, Faculty of Sciences \& IT, Al-Zaytoonah University of Jordan,

\section{ABSTRACT}

Amman, Jordan, email: dr.m.alia@ zuj.edu.jo

Face recognition has been largely used in biometric field as a security measure at air ports, passport verification, criminals' list verification, visa processing, and so on. Various literature studies suggested different approaches for face recognition systems and most of these studies have limitations with low performance rates. Eigenfaces and principle component analysis (PCA) can be considered as most important face recognition approaches in the literature. There is a need to develop algorithms and approaches that overcome these disadvantages and improve performance of face recognition systems. At the same time, there is a lack of literature studies which are related to face recognition systems based on EigenFaces and PCA. Therefore, this work includes a comparative study of literature researches related to Eigenfaces and PCA for face recognition systems. The main steps, strengths and limitations of each study will be discussed. Many recommendations were suggested in this study.

\section{Indexing terms/Keywords}

Face recognition, Eigenfaces, Eigenvectors, Eigenvalues, Principle Component Analysis (PCA).

\section{Academic Discipline And Sub-Disciplines}

Artificial Intelligence, Artificial Neural Networks, Image Processing.

\section{SUBJECT CLASSIFICATION}

Computer Science.

\section{TYPE (METHOD/APPROACH)}

Literary Analysis; Survey/Interview

\section{Council for Innovative Research}

\section{Peer Review Research Publishing System}

\section{Journal: INTERNATIONAL JOURNAL OF COMPUTERS \& TECHNOLOGY}

Vol 14 . No. 4

www.ijctonline.com, editorijctonline@gmail.com 


\section{INTRODUCTION}

Face recognition has a large number of applications such as: security, person verification, Internet communication, and computer entertainment. The problem of face recognition is still unsolved and offers a great challenge to researchers although research in face recognition has been conducted since 1960s. There is a significant progress in the area of face modeling and analysis techniques in the recent years. Many systems have been developed for face detection, tracking and recognition for security; need for identity verification in digital world; and the need for face analysis and modeling techniques in multimedia data management and computer entertainment. Recent advances in automated face analysis, pattern recognition, and machine learning have made it possible to develop automatic face recognition systems to address these applications. A face recognition system can be used to identify faces present in images and videos automatically. It can operate in either or both of two modes: face verification/authentication, and face identification/recognition. Face verification involves one-to-one match that compares a query face image against a template face image whose identity is being claimed. Face identification involves one-to-many matches that compares a query face image against all template images in the database to determine the identity of query face [1]. During the past few years, face recognition has received significant attention in the fields of image analysis and understanding [2]. Tolba, et al (2006) [3] provided an overview of face recognition techniques and its applications. They discussed the face databases which are used to test the performance of these face recognition algorithms [3]. Face recognition can be done in still image and video which has its origin in still image face recognition [4].

Eigenfaces for face recognition can implement an efficient, simple, and accurate face recognition system in a constrained environment such as a household or an office. The system does not depend on 3-D models of the structure of face (eyes, nose, mouth). Classification is performed using a linear combination of characteristic features (eigenfaces) [5]. There are many literature studies related to face recognition systems based Eigenfaces [6..12].

Principal Component Analysis (PCA) is a statistical method that have been used in image recognition. PCA can be used as feature extraction in face recognition to reduce large dimensionality of data space to smaller dimensionality of feature space when there is strong correlation between observed variables. PCA is used for prediction, redundancy removal, feature extraction and data compression. Applications (signal processing, image processing, system and control theory, communications) having linear models are suitable for PCA because PCA is a linear domain technique in biometrics. The PCA can be used for face recognition to express large one dimensional vector of pixels constructed from 2-D facial image into compact principal components of feature space (reduce multidimensional data to lower dimensions). This can be called eigenspace projection. Eigenspace is calculated by identifying eigenvectors of covariance matrix derived from a set of facial images (vectors) [13]. Many literature studies in the past related to face recognition based on PCA [14..23] Kuldeep and Madan (2013) [24] discussed the performance of face recognition techniques based PCA. They proposed a system which combines various parameters (distance classifier, applying histogram equalization, and selecting number of eigenfaces) into one face recognition system [24]. Whereas, Sasan Karamizadeh, et al (2013) [25] provided a detailed description of the PCA technique for face recognition. Other literature studies which are related to face recognition systems based on Eigenfaces with PCA [26..33].

Face recognition can be regarded as one of the most important topic in biometric studies. There is an important need to improve the performance of current face recognition approaches and algorithms. There is a lack of surveys which are related to face recognition systems based on EigenFace and PCA. Therefore, this work includes a comparative study on literature related to face recognition systems using EigenFace and PCA. This work is organized as follows: section 2 include literature studies related to face recognition based Eigenfaces and PCA. Section 3 describes in details the EigenFaces, Eigenvalues and Eigenvectors for face recognition. Section 4 includes description of PCA. Section 5 includes discussion and comparisons between literature studies. And finally, section 6 concludes this work.

\section{LITERATURE STUDIES}

\section{EigenFaces Studies}

Many techniques for face recognition using EigenFaces were suggested and discussed in the past. Moghaddam, et al (1998) [6] proposed technique for visual matching of images for face recognition. They proposed similarity measure based on Bayesian analysis of image differences. They model two classes of variation between two facial images: intra-personal (variations in appearance of same individual due to different expressions or lighting) and extra-personal (variations in appearance due to difference in identity). The probability density functions for each class are obtained from training data using an Eigenspace density estimation technique and used to compute a similarity measure based on a posteriori probability of membership in intra- personal class, which is used to rank matches in database [6].

And Raphael and Brian (1999) [8] designed of a real-time face recognition system to operate in less constrained environments. The system is capable of single scale recognition with an accuracy of $94 \%$ at 2 frames per second. They used Eigenfaces for face recognition.

Also, Zahid R., et al. (2008) [9] described an approach for face recognition that includes two steps: segmenting face region from an image by using an appearance based model; using eigenfaces for person identification for segmented face region. The efficiency lies on combined use of aforementioned techniques and in generation of appearance models which uses approach for shape and texture. Experiments have been performed on Cohn Kanade facial DB using 10 subjects for training and sever for testing purposes. They achieved face recognition rate up to $92.85 \%$ with and without facial expressions. Face recognition using PCA is fast and efficient to use, while the extracted appearance model can be further 
used for facial recognition and tracking under lighting and pose variations. This combination is simple to model and apply in real time [9].

And Sudhanshu, et al. (2009) [10] developed an approach to detect and identify of real time face recognition system with Graphical User Interface (GUI). This system takes a snap of a person from camera and store at a predefined place. The image face is treated as a two-dimensional recognition problem. Face images are projected on feature space that encodes the variation among the known face images. The feature space is defined the eigenfaces which are eigenvectors of faces. The Machine vision system designed have the feature that camera can rotate in horizontal and vertical direction [10].

Daniel Georgescu (2011) [11] implemented a real-time system for face detection and recognition in a video stream provided by a surveillance camera. Eigenfaces is used to solve recognition problem for two dimensional representations of faces using PCA. This system take snapshots which representing input images. These images are projected into a face space that defines variation for face images training set. Eigenfaces are used to define face space and represented eigenvectors of the set of faces. These Eigenfaces contribute in reconstruction of a new face image projected onto face space with a weight. The Euclidian distance is used to compare new image projection in this feature space with available projections of training set to identify person [11]. Finally, Tayal, et al, (2013) [12] proposed an Eigen based algorithm for face recognition from the database. This is typical task because face image changes with facial expression, age, viewpoint, illumination conditions and noise. At the same time, facial expression includes important information about mental, emotional and physical states of conversation.

\section{PCA Studies}

PCA is a classical feature extraction and data representation technique widely used in pattern and face recognition. But it has drawback of high computational especially for big size database. Jun-ying Gan, et al. (2005) [16] presented a method combined with the characteristics of PCA. This method is based on normalization of within-class average face image and has advantages of enlarging classification distance [16] between class samples. The method is based on computing average of faces training samples within class to normalize training samples. Fig. 1 shows this suggested method Eigenface space is used later. The average face of all training samples is used to normalize all samples to be compared under same conditions. Training and testing samples are projected into Eigenface space to get feature. Nearest Neighbor Distance (NND) is used in classification. They used ORL (Olivetti Research Laboratory) face database. Results achieved recognition rate equal $98 \%[16]$.

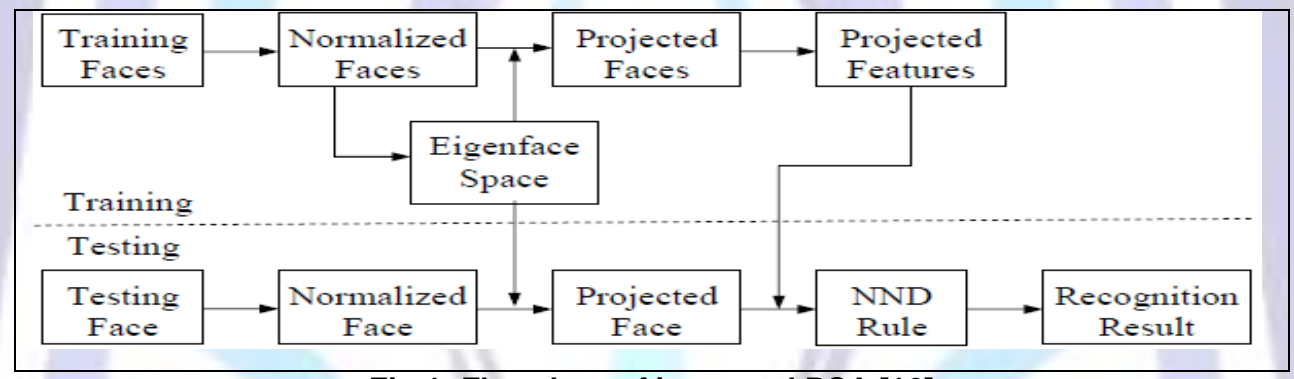

Fig 1: Flowchart of improved PCA [16]

Renke P. S. (2010) [17] proposed a methodology for improving face recognition rate. The method is tested using ORL database. Recognition rates for a database of 100 images shows promising results [17]. Fig. 2 and Fig3 show the detailed description of this algorithm.

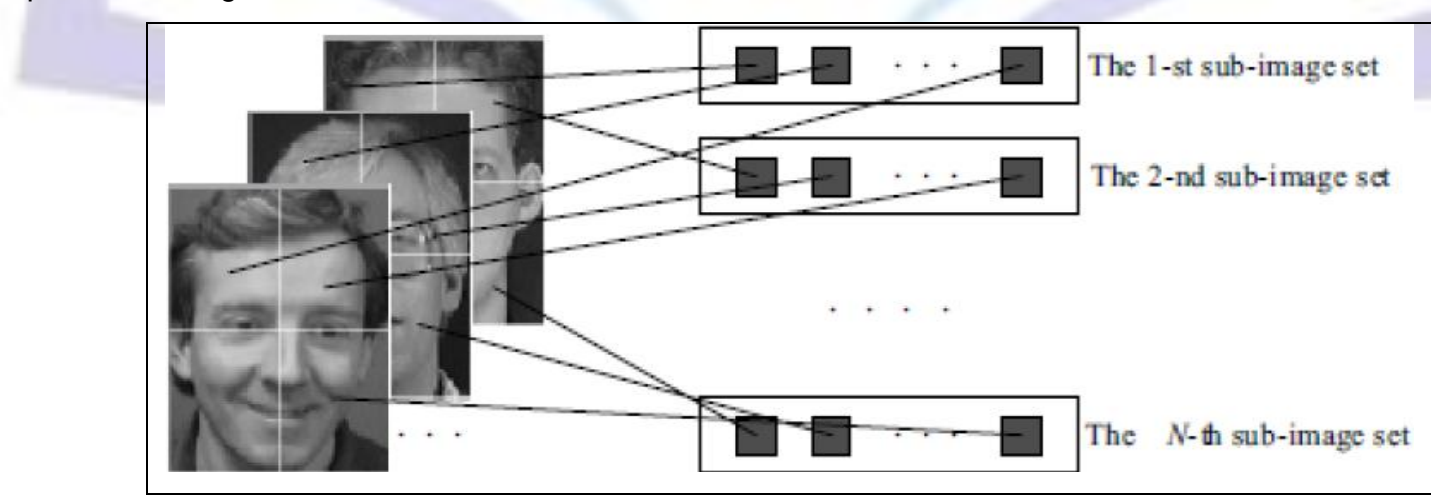

Fig 2: Construction of sub-image sets [17]

Hussein Rady (2011) [18] proposed face recognition system for personal identification/verification using PCA with different distance classifiers. The ORL face database is used to test system. Different classifiers (city-block distance; Euclidian distance; squared Euclidian Distance; and Squared Chebyshev distance) were used to match image of a person to a class obtained from training data. The Euclidian Distance Classifier produces a recognition rate higher than the City-Block Distance Classifier which gives a recognition rate higher than the Squared Chebyshev Distance Classifier. Fig.4 shows Schematic diagram of a face recognizer. 


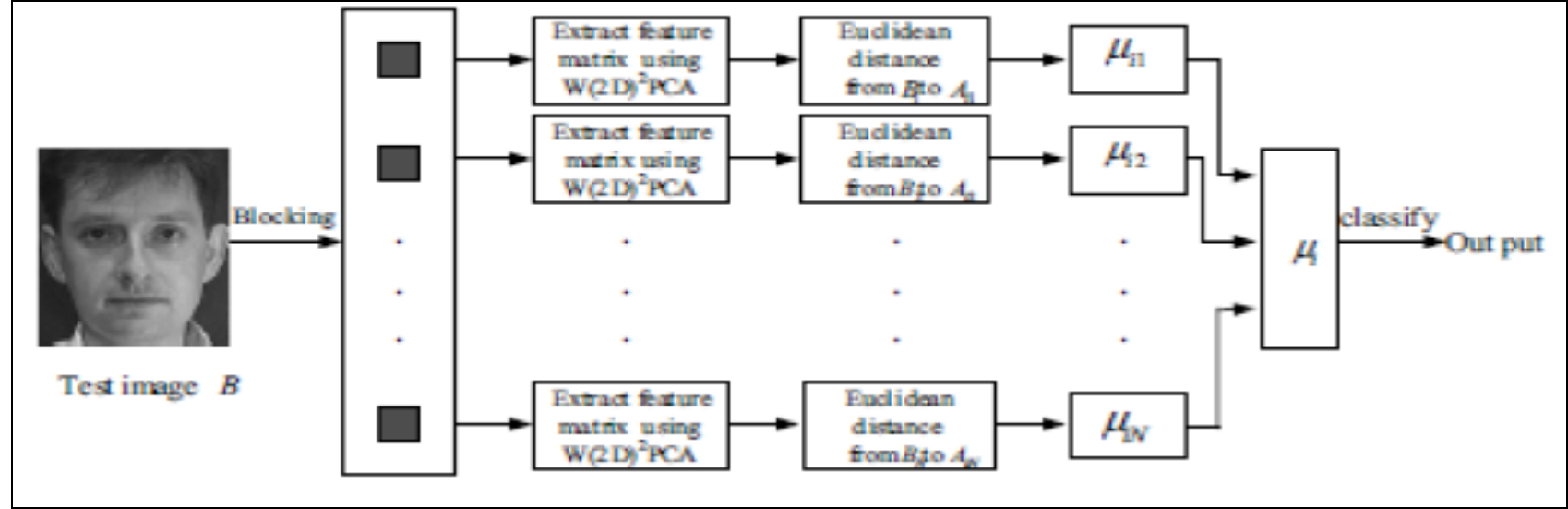

Fig 3: Processing of face image recognition [17]

At the same time, Vinay and Ashutosh (2012) [19] developed a face recognition system using an extended PCA algorithm to deal with the problem of orientation and lightening conditions present in original PCA. The preprocessing phase of the proposed algorithm emphasize the efficiency of the algorithm even when number of images per person or the orientation is very different [19].

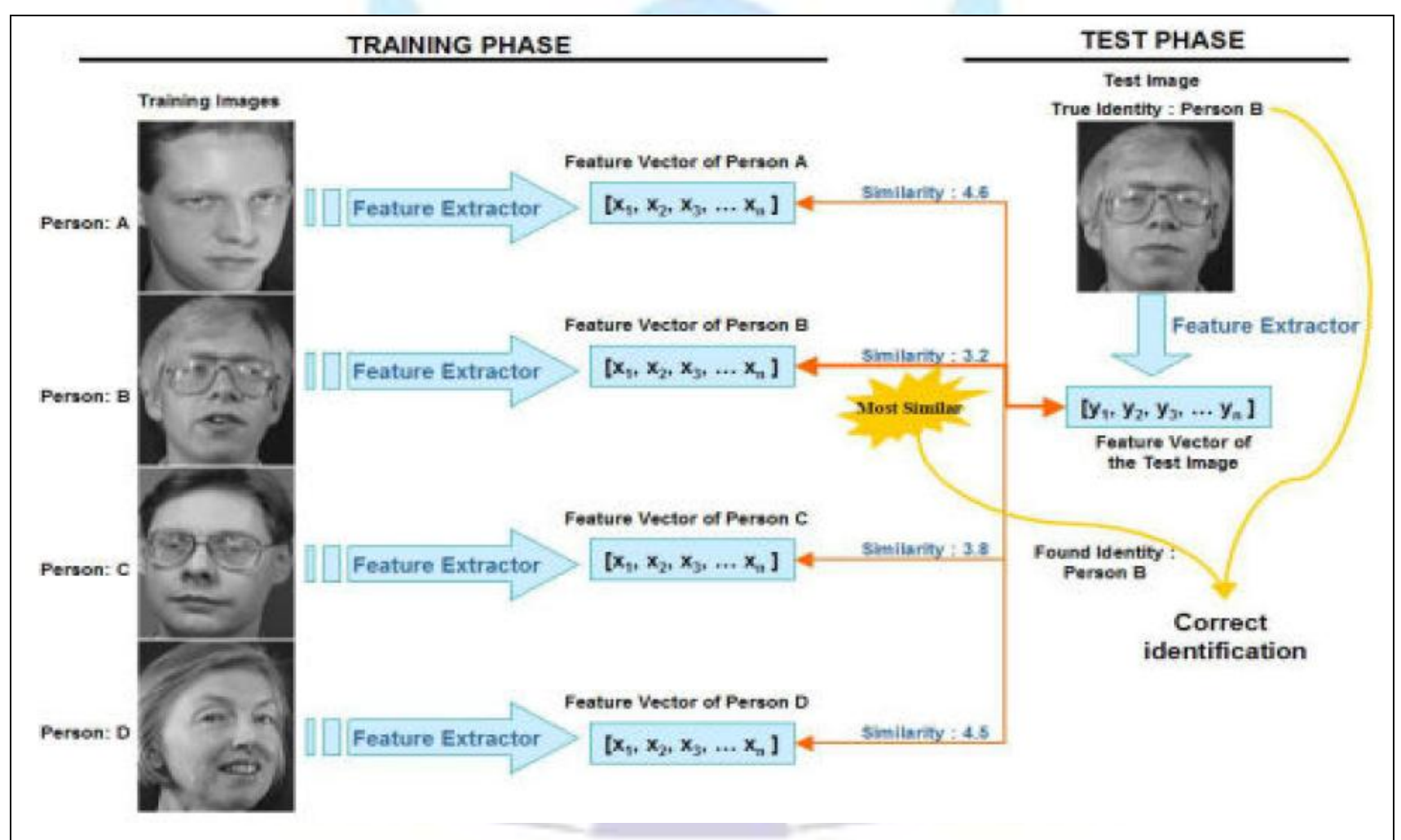

Fig 4: Schematic diagram of a face recognizer [18]

Manal A., et al. (2012) [20] conducted study to optimize the time complexity of PCA that does not affects the recognition performance. They minimized the participated eigenvectors which consequently decreases the computational time. A comparison is done to compare the differences between the recognition time in original and enhanced algorithms. The performance of the original and the enhanced proposed algorithm is tested on face 94 face database. Experimental results showed that the proposed enhanced algorithm reduced recognition time by $35 \%$.

Gunjan D.and Cyril Raj (2012) [21] conducted PCA for face recognition by projecting face image onto a feature space that spans the significant variations among known face images. The significant features are known as Eigen faces because they are the eigenvectors of the set of faces they do not correspond to the features (eyes, ears, and noses). The projection operation characterize an individual face by a weighted sum of Eigen faces features and so to recognize a particular face it is necessary only to compare these weights to those individuals. The results showed accuracy of singleface detection better than $92 \%$ with simple background and sufficient light source.

Marijeta and Dubravka (2012) [22] implemented face recognition system using the PCA based on an eigenfaces approach in which a small set of significant features are used to describe the variation between face images. They conducted many experimental results for different numbers of to verify the viability of the proposed method. They computed Euclidean Distance as recognition rate and the results vaeied from $77.5 \%$ to $97.5 \%$. 
And Abhishek and Saurabh (2012) [23] treated face recognition as a two-dimensional recognition problem and adopted Eigenface approach that uses PCA. Their system projected pre extracted face images onto a face space that encodes/represents best variation among known face images. Eigenface is used to define face space and these eigenfaces are eigenvectors of the set of faces. After matching with the present database, face will be categorized as known or unknown face. If the user is new to face recognition system then his template will be stored in database otherwise, matched against database templates.

\section{EigenFaces with PCA Studies}

The main challenge in face recognition systems is related to images taken in non ideal illumination conditions. There has been significant progress in improving the performance of computer-based face recognition algorithms over the last decade. Although algorithms have been tested and compared extensively with each other, there has been remarkably little work comparing the accuracy of computer-based face recognition systems with humans [29]. Quintiliano et, al. (2001) [26] conducted Eigenfaces and PCA for face recognition for poorly illuminated faces from Yale Face Database. The obtained recognition rates are above $72 \%$ [26].

And, Tsalakanidou, et al (2003) [27] developed face recognition technique based on the implementation PCA and the extraction of depth and colour eigenfaces (depth and colour information). They evaluated three approaches (colour, depth, combination of colour and depth) for face recognition and quantify the contribution of depth. They applied their suggested approach on XM2VTS database which contains $(720 \times 576)$ images of 295 persons each with 12images.

Nupoor M. Yawale (2013)[29] proposed a face recognition system based on Eigen faces. Face detection is to detect a human face in any given image and face recognition algorithm is to match it against a known face database. This face detection algorithm works by locating eyes in the image and the face recognition algorithm uses PCA to calculate eigenvalues and eigenvectors of the face images. This paper discusses a generic framework for the face recognition system, and the variants that are frequently encountered by the face recognizer. It also discusses the requirements of a robust face recognition system. Also face detection algorithm based on eye-analogue segmentation and recognition algorithm based on Eigen-space have been developed by us. And Rajib and Debotosh (2013) [30] used Eigenfaces using PCA for face recognition. They discussed the features that are important for classification to reduce the dimensionality of training set. Their experimental results have shown that face images can be successfully recognize with the rate of $96 \%$.

Rajkiran and Vijayan (2004)[33] proposed technique for face recognition by dividing the face images into smaller sub images. Then they applied PCA approach to each of these sub images. They evaluated the accuracy of conventional PCA and proposed PCA method under conditions of varying expression, illumination and pose using standard face databases. They used two image databases (UMIST and Yale.Their proposed algorithm has an improved recognition rate with large variations in lighting direction and facial expression when compared with conventional PCA [33]

\section{FACE RECOGNITION USING EIGENFACES}

Face recognition can be categorized into three approaches: Feature based; Holistic; and Hybrid. In holistic approach, the input data into face detection system is a whole face region. Many methods of holistic approach: Eigenfaces; probabilistic Eigenfaces; fisherfaces; support vector machines; nearest feature lines (NFL); and independent component analysis. These approaches are based on PCA techniques to simplify a dataset into lower dimension with maintaining the characteristics of dataset. In feature based approaches, local face features (nose, eyes) are segmented and used as input data for the structural classifier. Many methods related to this approach: pure geometry; dynamic link architecture; and hidden Markov. The hybrid approach perceives local feature and whole face. There are modular Eigenfaces; hybrid local feature; shape normalized; component based methods in hybrid approach [4].

\section{EigenFaces}

Facial recognition is discriminating input image into several classes (persons). Eigenfaces are a set of standardized face component (eigenvectors) based on statistical analysis (covariance matrix) of various face images (high dimensional vector that represents faces of humans). The human face can be represented by linear combination of Eigenface images (matrix). The eigenvector represents component. A matrix has a set of eigenvectors that represent the principal components of the matrix.

A new face image is projected onto face space (set of Eigenface features in face recognition system) by multiplying the difference between the image and average. The result is multiplied by each eigenvector. The eigenvectors corresponding to these features are weighted. The sum of these weights is a good representation of a given face. Euclidean distance is calculated between the mean adjusted input image and projection onto face space. Euclidean distance taken from each face class determines the class that best matches the input image [4][20][22][23]

The Eigenfaces can be extracted out of original image by PCA [4]. PCA transforms each original image of training set into Eigenface. PCA can reconstruct any original image from training set by combining Eigenfaces. Each Eigenface represents certain features of face that may or may not be present in original image. The share of corresponding Eigenface in the sum of Eigenfaces should be greater if the feature is present in original image to a higher degree. The corresponding Eigenface should contribute a smaller part to sum of Eigenfaces if the particular feature is not present in original image. Weighted sum is computed of all Eigenfaces to reconstruct original image from Eigenfaces. The reconstructed original image is equal to sum of all Eigenfaces with each Eigenface having a certain weight. This weight specifies to what degree the specific Eigenface is present in original image. We can reconstruct original images from Eigenfaces if we use all Eigenfaces extracted from original images. The reconstructed image is an approximation of original image if we use only 
part of Eigenfaces. Similar faces are faces which have similar weights. Similar faces possess similar Eigenfaces to similar degrees (weights). Images could be grouped to clusters if one extracts weights from all images available. Fig.5 describes face recognition algorithm using Eigenfaces [4][20][22][23].

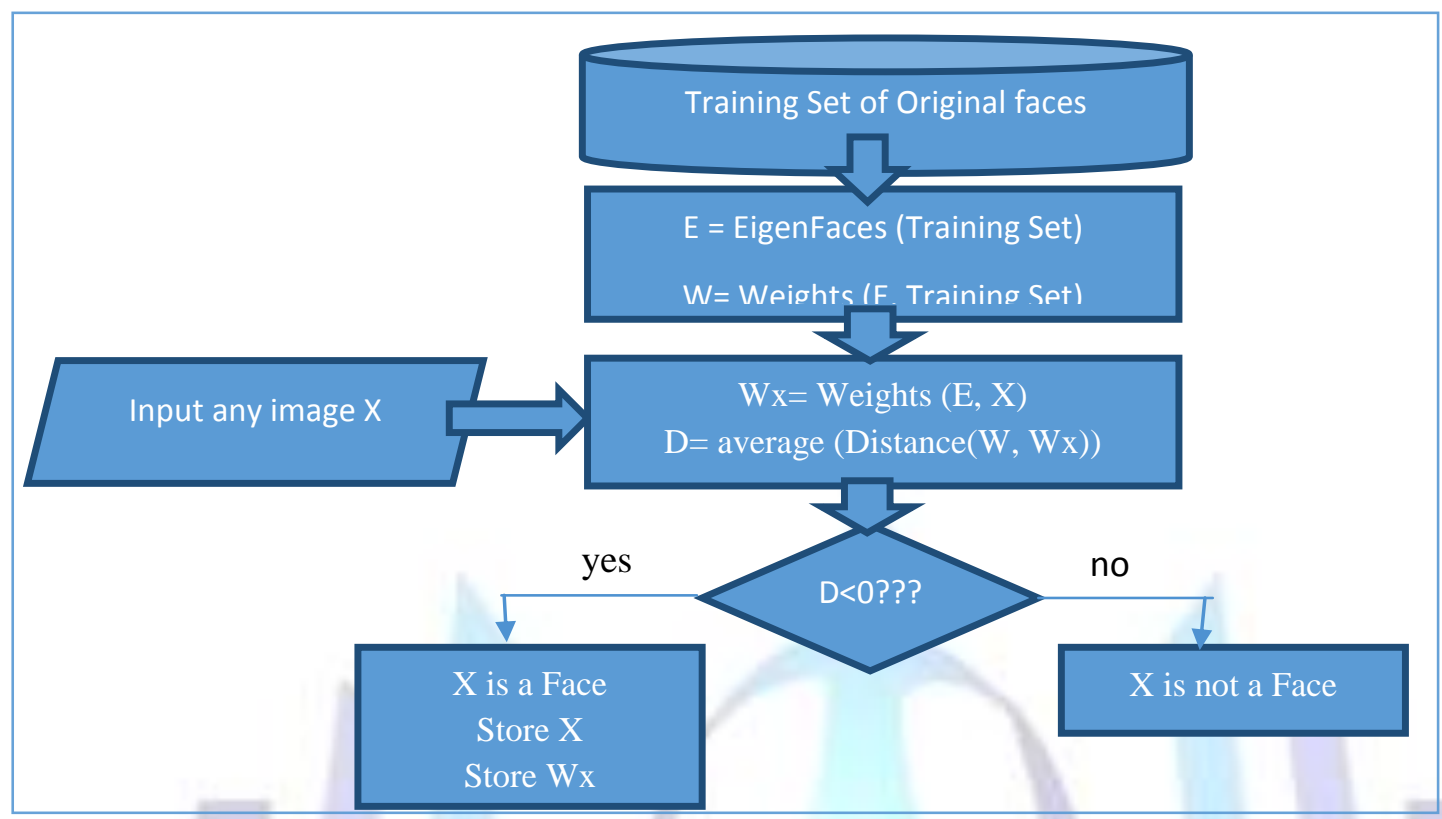

Fig 5: Face Recognition Using EigenFaces [4]

\section{Eigenvectors and Eigenvalues}

An eigenvector of matrix is a vector that if is multiplied with this matrix, the result is an integer multiple of that vector. This integer value is named eigenvalue of eigenvector. Eigenvectors can be determined only for square matrices. There are $n$ eigenvectors (and their eigenvalues) in $\mathrm{n} \times \mathrm{n}$ matrix. All eigenvectors are perpendicular. This relationship can be described by the equation[4][20][22][23][24]:

$\mathrm{M} \times \mathrm{u}=\lambda \times \mathrm{u}$

Where, $u$ : eigenvector of matrix $M$, and $\lambda$ : corresponding eigenvalue.

\section{Mathematical Foundations of Eigenface}

The mathematical equations related to face recognition using Eigenfaces as described in details as follows: [4][20][22] [23][24][25][28][32]:

1. Let $\Gamma i(x, y)$ be a face image with $m \times n$ array of intensity values. Prepare the training set of images $(\Gamma i)$ from faces $\{\Gamma 1$, $\Gamma 2, \Gamma i 3, \ldots, \Gamma \mathrm{M}\}$.

2. Calculate the average matrix of face $(\psi)$.

$$
\Psi=\frac{1}{M} \sum_{n-1}^{M} \Gamma_{n}
$$

3. Subtract the mean $(\Psi)$ from original faces $(\Gamma i)$, and store result in pi.

$$
\Phi_{i}=\Gamma_{i}-\Psi
$$

4.Calculate the covariance matrix $(C)$ to represent the scatter degree of all feature vectors related to the average vector as the following equation:

$$
C=\frac{1}{M} \sum_{n-1}^{M} \Phi_{n} \Phi_{n}^{T}
$$

5. Calculate Eigenvectors (Eigenfaces) (ui) and corresponding eigenvalues $(\lambda i)$ from covariance matrix $(C)$.

6. Normalize eigenvectors to be unit vectors of length 1 .

7. Sort the eigenvector according to their eigenvalues from high to low. Select principal components(feature vector): chose just M' (which have highest eigenvalues) from M Eigenfaces (ui). The higher eigenvalue means more characteristic features of face does the eigenvector describe. At the same time omitted the Eigenfaces with low Eigenvalues because they explain only small part of faces' features.

8. The algorithm training phase is finished after determining the $\mathrm{M}^{\prime}$ eigenfaces (ui) 


\section{PRINCIPAL COMPONENT ANALYSIS (PCA)}

PCA is a statistical analysis method based on Karhunen Loeve's transformation. PCA can reduce the high dimensionality of face image (vector project) to low-dimensional vector space. This is done by a specific feature vector matrix. Finally, we can reconstruct the corresponding original high-dimensional vector. PCA can retain its key identifying information [31][32].

\section{PCA Training Algorithm}

The PCA training algorithm for face recognition can be summarized by the following steps [4][31][4][31] [18][20][32]:

1. Each face in training set of images is represented using s-dimension vector.

2. Create the transformation matrix using the training images.

3. The training images are projected onto the matrix columns. PCA finds t-dimension subspace whose basis vectors correspond to maximum variance direction in original image space. Where t subspace is lower dimensional $(\mathrm{t}<<\mathrm{S})$

4. PCA vectors are defined as eigenvectors of scatter matrix if image elements are considered as random variables.

5. The Eigenface algorithm uses PCA for dimension reduction to find vectors which best account for distribution of face images within entire image space.

6. These vectors define subspace (face space) of face images.

7. Find a set of weights that describes contribution of each vector in face space, all faces in training set are projected onto face space.

\section{PCA Testing Algorithm}

The PCA algorithm for identifying/testing any face image can be summarized by the following steps [4][31] [18][20][32]:

Each test image should be mean centered

Projection of test image onto same face space (subspace) Eigenspace as defined during the training phase. This is done to obtain the corresponding set of weights.

Comparing weights of test image (projected image) with the set of weights of faces in training set (eigenspace). This is done identify the test face image.

Images are compared with similarity measures.

The training image that is closest to the test image will be matched as used to identify.

PCA vectors are defined as eigenvectors of scatter matrix ST as the following equation [4]

$$
\mathrm{S}_{\mathrm{T}}=\sum_{\mathrm{i}=1}^{\mathrm{N}}\left(\mathrm{x}_{\mathrm{i}}-\mu\right)\left(\mathrm{x}_{\mathrm{i}}-\mu\right)^{\mathrm{T}}
$$

\section{COMPARATIVE ANALYSIS}

Many studies related to face recognition systems were suggested in the past few years. In this paper, a comparative analysis of exiting face recognition methods was presented to examine the performances of these studies in terms of face recognition rates. It is important to suggest method to reduce dimensionality, feature extraction and classification for face recognition system. Many of these provided studies [6..12] were based on Eigenfaces. Other studies were based on PCA [14..23]. Finally other studies [26..33] were based on Eigenfaces with the using of PCA. Each one of these studies were focused essentially on performing face recognition process with good performance measures. But each with some limitations related to required time and memory, poor recognition rate, inability to recognize faces with different pose and illumination, and so on. There is always a need to improve the algorithms and approaches related to face recognition systems by studying the advantages and limitations of current algorithms and approaches.

At the same time, there is a need for general data base with large number of persons that includes many poses and illuminations. Because this is essential for better evaluations of face recognition approaches. Many large available databases for faces have been collected during the past years (FERET[34], Cohn-Kanade [35], Extended Yale DB[36], ORL DB [37], CASIA [38], and so on).

Most of literature studies that related to face recognition systems made decisions according to distance measure. These approaches were based on projecting images to lower dimension feature space. Other literature studies based on Eigenfaces were concerned with one pose. In order to estimate the actual face, we need for the same person a collection of different pictures of different angles and emotions. Better recognition rate will be found to recognize the person if we give more sample pictures of that person.

Table 1 shows the characteristics of many of literature studies which related to face recognition systems. These characteristics include: approach used, database used, sample size of training and testing images, recognition rate, and required time.

From Table 1, we can note that, most of the literature studies were based on Eigenfaces with PCA. Each one of these studies was adopt different database for face recognition approach. They used different image dimensions. They used 
different benchmarking approach to test the algorithm performance. The recognition rates are different and ranged between 72 and 94 . Also, just 2 studies were calculate the required time and it is ranged between 4.54 and 35 . Few literature researches were based on real time [8][10][11]. Finally, these methods have been successfully applied for face recognition but each with its advantages and disadvantages.

\section{Problems and Lessons learned}

During the analysis of the literature related to face recognition which were based on Eigenface and PCA, we noticed the following points:

1. Most of literature studies were based on still images and very few studies were based on video. Video based face recognition systems showed good results in controlled environments.

2. Recognition of faces from video sequence is a challenging problem because video is of low quality and images are small.

3. One of the difficulties in literature studies is how to obtain database with good quality gallery images.

4. It is difficult to build robust face recognition because there are many challenges such as: $100 \%$ recognition rate, algorithm complexity, illumination, pose, and recognition in outdoor imagery. We presented review of approaches proposed in literature to solve these. Many of these problems remain to be unsolved such as: accurate pose estimation is hard. .Lack of face recognition studies which consider illumination and pose problems.

5. Another difficulty related to face recognition systems is recognition of a person from images taken before many years.

6. Many literature studies has good performance but they were based on small image size such as: $(128 \times 128),(96 \times 84)$ or $(30 \times 25)$ which restricts its application.

7. There are few evaluations and benchmarking techniques of face recognition approaches and algorithms. This is lead to significant impact on progress in proposition of face recognition algorithms.

This lower performance of face recognition approaches in literature has motivated to suggest new effective face recognition method that take the following conditions in consideration:

1. Develop an algorithm that is based on database of face images with different face pose and illumination conditions.

2. Develop real time face recognition algorithm that take a sequence of images (frames) from digital video camera.

3. Develop algorithm that is not affected by the background of the face image.

4. Develop algorithm that take the advantages of Eigenfaces with the advantages of other artificial intelligence algorithm such as artificial neural networks (ANN) as a good classifier.

5. Develop an algorithm that based on medium image dimension size and not very small.

Table1: performance analysis of face recognition studies

\begin{tabular}{|c|c|c|c|}
\hline Research & Method/Approach & Database & Recog. Rate \\
\hline [6] (1998) & EigenFaces, Bayesian analysis & FERET DB [34] & $\begin{array}{ll}---- \\
\end{array}$ \\
\hline [8] (1999) & EigenFaces & & $\begin{array}{l}94 \% \\
\text { Real Time }\end{array}$ \\
\hline [9] (2008) & Segmenting, EigenFaces,PCA & $\begin{array}{l}\text { Cohn Kanade facial DB [35] } \\
10 \text { subjects for training }\end{array}$ & $92.85 \%$ \\
\hline [10] (2009) & $\begin{array}{l}\text { EigenFaces, Euclidean Distance, } \\
\text { PCA }\end{array}$ & & Real Time \\
\hline [11] (2011) & EigenFaces, PCA & $\begin{array}{l}\text { Libor Spacek's Collection of Facial Images } \\
\text { [IMG2] }\end{array}$ & Real Time \\
\hline [12] (2013) & EigenFaces, Euclidean Distance, & $\begin{array}{l}\text { Yale DB-Extended Yale DB[36] } \\
\text { FERET DB }(180 \times 200)\end{array}$ & $\begin{array}{l}\text { Time }=4.54 \\
\text { sec }\end{array}$ \\
\hline [16] (2005) & PCA, Eigenface, NND & $\begin{array}{l}\text { ORL [37]. } 400(112 \times 92) \text { face images, } 256 \\
\text { gray }\end{array}$ & $98 \%$ \\
\hline [17] (2010) & PCA and DCT & $\begin{array}{l}\text { ORL[37].100 }(92 \times 112) \text { face images, } 256 \\
\text { gray }\end{array}$ & $92.60 \%$ \\
\hline [18] (2011) & $\begin{array}{l}\text { PCA, (city-block distance; Euclidian } \\
\text { distance; squared Euclidian Distance; }\end{array}$ & Olivetti Research Lab (ORL)[37] & $\begin{array}{l}93.3 \% \\
95.2 \%\end{array}$ \\
\hline
\end{tabular}




\begin{tabular}{|c|c|c|c|}
\hline & Squared Chebyshev distance) & & \\
\hline [19] (2012) & Improved PCA & $\begin{array}{l}\text { FERET DB- } 110 \quad(56 \times 384) \text { images, } 10 \\
\text { persons each with } 11 \text { images }\end{array}$ & --------- \\
\hline [20] (2012) & Optimized PCA & $\begin{array}{l}\text { face94 face DB: }(180 \times 200) \text { image for } 28 \\
\text { persons each with } 6 \text { in test DB }\end{array}$ & $\begin{array}{l}\text { Rec } \\
\text { time }=35 \%\end{array}$ \\
\hline [21] (2012) & PCA, Eigenfaces & & $92 \%$ \\
\hline [22] (2012) & $\begin{array}{l}\text { PCA, EigenFaces } \\
\text { Euclidean Distance, }\end{array}$ & $\begin{array}{l}\text { ORL DB of faces. Training DB: } 190 \\
(92 \times 112) \text { images of } 38 \text { persons each with } 5 \\
\text { images. Test DB : } 40 \text { images }\end{array}$ & $\begin{array}{l}\text { between } \\
77.5 \% \text { and } \\
97.5 \%\end{array}$ \\
\hline [23] (2012) & PCA, EigenFaces & & \\
\hline [26] (2001) & EigenFaces, PCA & Yale Face Database & $>72 \%$ \\
\hline
\end{tabular}

\section{CONCLUSION}

Different approaches for face recognition systems based on EigenFaces and PCA were briefly listed and discussed in this work. We have been considered 3 types of face recognition studies (Eigenfaces; PCA; and Eigenfaces with PCA) and discussed their characteristics. Also, we discussed the Eigenfaces algorithm and PCA algorithm for face recognition. Comparisons between different literature studies for face recognition systems based on Eigenfaces and PCA were discussed also.

We provided a summary of literature studies for face recognition systems with different: databases; number of training samples; number of testing samples; image size; classifiers; and performance measures (such as recognition rate, error, or required time). Many problems in literature studies were discussed. And finally, lessons and recommendations based on these problems were suggested in this work. In our future research, an improved real time face recognition system will be suggested and developed based on Eigenfaces algorithm.

\section{ACKNOWLEDGMENTS}

Our appreciates and thanks to AIZaytoonah University of Jordan- Amman-Jordan for supporting the scientific research.

\section{REFERENCES}

[1] Stan Z. Li and Anil J. K. 2005. Handbook of Face Recognition, Springer Science $\square$ Business Media, Inc.

[2] Zhao W., et al. 2003. Face Recognition: A Literature Survey, ACM Computing Surveys, Vol.35, No.4, pp. 399-458, December 2003.

[3] Tolba A. S., et al. 2006. Face Recognition: A Literature Review, International Journal of Information and Communication Engineering, Vol.2,No.2, pp:89-103, 2006.

[4] Shariful I. F. and Sabbir A. 2008. Literature Survey Of Automatic Face Recognition System And Eigenface Based Implementation, Thesis, Department of CS and Engineering, BRAC University, Bachelor of Science in CS and Eng., August 2008.

[5] Turk M., Pentland A. 1991. Eigenfaces for Recognition. Journal of Cognitive Neuroscience. Vol.3, No.1, pp:71-86.

[6] Baback M., et al. 1998. Beyond Eigenfaces: Probabilistic Matching for Face Recognition, 3rd IEEE Int'I Conference on Automatic Face \& Gesture Recognition, Nara, Japan, April 1998.

[7] Thomas Heseltine, et al. 2002. Evaluation of image pre-processing techniques for eigenface based face recognition, Proceedings of the Second International Conference on Image and Graphics, SPIE vol. 4875, pp. 677-685 (2002)

[8] Raphael C. and Brian L., 1999. Real-Time Face Recognition using Eigenfaces, 1999.

[9] Zahid R., et al., 2008. Shape Invariant Recognition of Segmented Human Faces using Eigenfaces, Multitopic Conference, 2008. INMIC 2008. IEEE International, Date 23-24 Dec. 2008.

[10] Sudhanshu T., et al. 2009. Face Recognition Machine Vision System Using Eigenfaces, International Journal of Recent Trends in Engineering, Vol.2, No.2, pp:1-3, November 2009.

[11] Daniel G., 2011. A Real-Time Face Recognition System Using Eigenfaces, Journal of Mobile, Embedded and Distributed Systems, Vol.3, No.4, 2011, ISSN 2067 - 4074.

[12] Tayal, et al, Face Recognition using Eigenface, International Journal of Emerging Technologies in Computational and Applied Sciences, Vol.3, No.1, pp:50-55, Dec.12-Feb., 2013. 
[13] Turk M.A. and Pentland A.P., 1991. Face Recognition Using Eigenfaces, IEEE Conf. on Computer Vision and Pattern Recognition, pp. 586-591, 1991.

[14] Bruce A. D., et al. 2003. Recognizing faces with PCA and ICA, Computer Vision and Image Understanding 91 (2003) 115-137.

[15] Taranpreet S. R., 2005. Face Recognition Based on PCA Algorithm, Special Issue of International Journal of Computer Science \& Informatics (IJCSI), ISSN (PRINT) : 2231-5292, Vol.2, Issue.1, 2, 2005.

[16] Jun-ying Gan, et al. 2005. A Method For Improved PCA in Face Recognition, International Journal of Information Technology Vol.11, No.11, 2005.

[17] Renke P. S., Automatic Face Recognition using Principal Component Analysis with DCT, Journal of Electronicsl and Communication Engineering (IOSR-JECE) ISSN: 2278-2834-, ISBN: 2278-8735, PP: 01-07 www.iosrjournals.org

[18] Hussein R., 2011. Face Recognition using Principle Component Analysis with Different Distance Classifiers, IJCSNS International Journal of Computer Science and Network Security, VOL.11 No.10, October 2011.

[19] Vinay R. and Ashutosh G., 2012. Improved PCA Algorithm for Face Recognition, World Applied Programming, Vol.2, Issue.1, January 2012. 55-59, Special section for proceeding of International e-Conference on Computer Engineering (leCCE).

[20] Manal A., et al. 2012. Optimizing Face Recognition Using PCA, International Journal of Artificial Intelligence \& Applications (IJAIA), Vol.3, No.2, pp:23-31, March 2012.

[21] Gunjan D.and Cyril Raj, 2012. An Efficient Method For Face Recognition Using Principal Component Analysis (PCA), International Journal of Advanced Technology \& Engineering Research, Vol.2, Issue.2, March 2012, pp:23-29.

[22] Marijeta S. and Dubravka J., 2012. Face Recognition Using Eigenface Approach*, Serbian Journal Of Electrical Engineering, Vol. 9, No.1, February 2012, pp:121-130, DOI: 10.2298/SJEE1201121S.

[23] Abhishek S. and Saurabh K., 2012. Face Recognition Using PCA and Eigen Face Approach, thesis, Bachelor of Technology, Department of Computer Science and Engineering, National Institute of Technology Rourkela, Rourkela769008, India, 2012.

[24] Kuldeep Singh S. and Madan L., 2013. Comparative Analysis of PCA-based Face Recognition System using different Distance Classifiers, International Journal of Application or Innovation in Engineering \& Management (IJAIEM), www.ijaiem.org Email: editor@ijaiem.org, Vol.2, Issue.7, July 2013, ISSN 2319 - 4847.

[25] Sasan Karamizadeh, et al. 2013. An Overview of Principal Component Analysis , Journal of Signal and Information Processing, 2013, 4, 173-175 doi:10.4236/jsip.2013.43B031, August 2013 (http://www.scirp.org/journal/jsip)

[26] P. Quintiliano, et al. 2001. Practical Procedures to Improve Face Recognition Based on Eigenfaces and Principal Component Analysis, Applied Problems In Systems Of Pattern Recognition And Image Analysis, Pattern Recognition And Image Analysis, Vol. 11, No. 2, 2001.

[27] F. Tsalakanidou, et al. 2003. Use of depth and colour eigenfaces for face recognition, Pattern Recognition Letters 24 (2003) 1427-1435.

[28] Sezin KayMak, 2003. Face Detection, Recognition and Reconstruction Using EigenFaces, EE574 Detection and Estimation Theory, Class Presentation, 2003, Department of Electrical and Electronic Engineering.

[29] Nupoor M. Yawale, Face Detection and Recognition using Eigen Faces by using PCA, Int.J.Computer Technology \& Applications, Vol.4, No.1, pp:126-129.

[30] Rajib S. and Debotosh B., 2013. Face Recognition Using EigenFaces, International Journal of Emerging Technology and Advanced Engineering, Vol.3, Issue.5, May 2013, pp:90-93.

[31] C. Han, 2009. Modular PCA Face Recognition Based on Weighted Average". Modern Applied Science, Vol.3, No.11, November 2009.

[32] Arjun V. Mane, et al. 2010. Human Face Recognition Using Superior Principal Component Analysis (SPCA), International Journal of Computer Theory and Engineering, Vol. 2, No. 5, pp:688-691, October, 2010, $1793-8201$.

[33] Rajkiran G. and Vijayan K., 2004. An improved face recognition technique based on modular PCA approach, Pattern Recognition Letters, vol.25, pp: 429-436, 2004, Elsevier.

[34] The Facial Recognition Technology (FERET) Database, http://www.itl.nist.gov/iad/ humanid/feret/feret_master.html

[35] Cohn-Kanade AU-Coded Expression Database, http://www.pitt.edu/ emotion/ck-spread.htm

[36] The Extended Yale Face Database B, http://vision.ucsd.edu/ leekc /ExtYaleDatabase/ExtYaleB.htm[37]

[37]The ORL Database of Faces, Cambridge University Computer Laboratory, http://www.cl.cam.ac.uk/ research/dtg/attarchive/facedatabase.html, 2002 AT\&T Laboratories Cambridge. 
[38] CASIA Face Image Database Version 5.0, National Laboratory of Pattern Recognition (NLPR) | Institute of Automation, Chinese Academy of Sciences(CASIA), 2010 TNT Group, http://biometrics.idealtest.org/dbDetailForUser.do?id=9

\section{Author' biography with Photo}
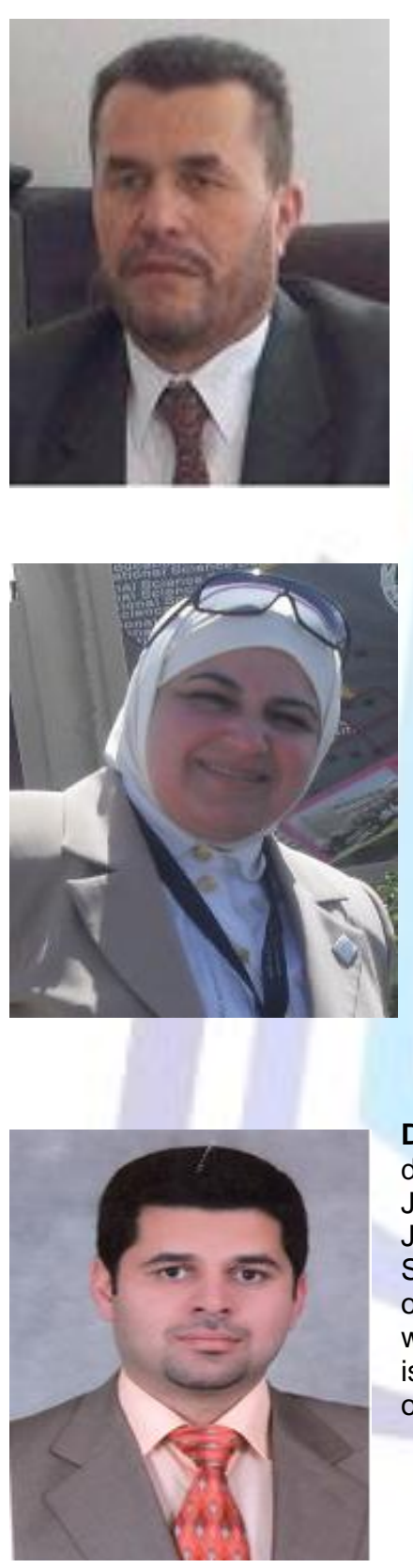

Dr. Abdelfatah A. Tamimi has been a member of the Faculty of Science and Information Technology at Al-Zaytoonah University since 1996, where he held different positions including the Dean of the Faculty and the Chair of the Department of Computer Science. He received his Ph.D. in computer science from the City University of New York, NY, USA; his Master's degree in computer science from Montclair State University, NJ, USA; and his Bachelor's degree in mathematics from Jordan University, Amman, Jordan. In addition to his research and teaching experience, he has a 15 year experience in information technology design, development and implementation in United States companies

Dr. Omaima N. A. Al-Allaf is an Assistant Professor at Basic Sciences department, Faculty of Sciences and IT, AL-Zaytoonah University of Jordan, Amman-Jordan (from 15 Feb. 2009). She received her B.Sc. in Computer Science from Dept. of CS, Faculty of Sciences, University of Mosul (1990-1994), and received her M.Sc. from Dept. of CS, Faculty of Computer \& Mathematical Sciences, University of Mosul (1997-1999), Mosul, Iraq. She worked as Teacher B in Applied Science University (Private)-Amman-Jordan from 2000 to 2004. She received her PhD in CIS from the Department of CIS, AABFSAmman-Jordan (2004-2008). During 2004-2008, she worked as a part time lecturer in the AABFS. Her research interests are in the fields of: artificial neural networks and image processing (recognition and compression), web engineering, software process models.

Dr. Mohammad Alia is an associate professor at the Computer Information Systems department, Faculty of Science and Information Technology, Al-Zaytoonah University of Jordan. He received the B.Sc. degree in Computer Science from Al-Zaytoonah University, Jordan, in 2000. He obtained his Ph.D. degree in Computer Science from University Science of Malaysia, in 2008. During 2000 until 2004, he worked at Al-Zaytoonah University of Jordan as an instructor of Computer Sciences and Information Technology. Then, he worked as a lecturer at Al-Quds University in Saudi Arabia from 2004 to 2005. Currently he is working as the Chair of Computer Information Systems dept. at Al Zaytoonah University of Jordan. His research interests are in the field of Cryptography and Network Security. 